\title{
Systems genetics and genome-wide association approaches for analysis of feed intake, feed efficiency, and performance in beef cattle
}

\author{
M.H.A. Santana ${ }^{1}$, M.C. Freua ${ }^{1}$, D.N. Do ${ }^{2}$, R.V. Ventura ${ }^{1,3}$, \\ H.N. Kadarmideen ${ }^{4}$ and J.B.S. Ferraz ${ }^{1}$ \\ ${ }^{1}$ Departamento de Medicina Veterinária, \\ Faculdade de Zootecnia e Engenharia de Alimentos, \\ Universidade do Estado de São Paulo, Pirassununga, SP, Brasil \\ ${ }^{2}$ Department of Animal Science, McGill University, \\ Sainte-Anne-de-Bellevue, Quebec, Canada \\ ${ }^{3}$ Centre for Genetic Improvement for Livestock, \\ University of Guelph, Guelph, Ontario, Canada \\ ${ }^{4}$ Section for Animal Welfare and Disease Control, \\ Faculty of Health and Medical Sciences, \\ University of Copenhagen, Frederiksberg, Denmark \\ Corresponding author: M.H.A. Santana \\ E-mail: miguel-has@hotmail.com
}

Genet. Mol. Res. 15 (4): gmr15048930

Received June 29, 2016

Accepted August 9, 2016

Published October 17, 2016

DOI http://dx.doi.org/10.4238/gmr15048930

Copyright (C) 2016 The Authors. This is an open-access article distributed under the terms of the Creative Commons Attribution ShareAlike (CC BY-SA) 4.0 License.

\begin{abstract}
Feed intake, feed efficiency, and weight gain are important economic traits of beef cattle in feedlots. In the present study, we investigated the physiological processes underlying such traits from the point of view of systems genetics. Firstly, using data from 1334 Nellore (Bos indicus) cattle and 943,577 single nucleotide polymorphisms (SNPs), a genome-wide association analysis was performed for dry
\end{abstract}


matter intake, average daily weight gain, feed conversion ratio, and residual feed intake with a Bayesian Lasso procedure. Genes within 50-kb SNPs, most relevant for explaining the genomic variance, were annotated and the biological processes underlying the traits were inferred from Database for Annotation, Visualization and Integrated Discovery (DAVID) and Kyoto Encyclopedia of Genes and Genomes (KEGG) databases. Our results indicated several putative genomic regions associated with the target phenotypes and showed that almost all genomic variances were in the SNPs located in the intergenic and intronic regions. We further identified five main metabolic pathways related to ion transport, body composition, and feed intake control, which influenced the four phenotypes simultaneously. The systems genetics approach used in this study revealed novel pathways related to feed efficiency traits in beef cattle.

Key words: Dry matter intake; Feed intake; Performance traits; Residual feed intake; Variance partitioning

\section{INTRODUCTION}

The importance of feed in economical production of livestock has been long recognized. Feed costs typically represent the largest share of variable costs affecting the long-term profitability of cattle feedlot operations (Anderson et al., 2005). In addition, when considering sustainable intensification, cattle finished in confinement might offer an opportunity to increase the production using less land. Moreover, it could be an economically sound development strategy to reduce greenhouse gas emissions, particularly when reducing the reliance on pasture for finishing animals and when the use of by-products in animal diets are favorable (Havlík et al., 2014). Thus, feed intake, weight gain, and feed efficiency - the three metrics required for optimizing the resource use, together with meat quality are important variables to facilitate the improvement of the overall economic and environmental sustainability of livestock production systems (Nkrumah et al., 2007).

Several studies have used genomic information for understanding the biological processes underlying these production traits in beef cattle (Moore et al., 2009; Rolf et al., 2012). Genome-wide association studies (GWAS) with dense panels of genetic markers have potential to identify the genomic regions that govern such phenotypes (Rolf et al., 2012). More recently, systems genetics (SG) has been proposed as an ideal approach for unifying phenotypic and genotypic databases to get a single conceptual mechanistic view on how phenotypes emerge as an outcome of long and complex dynamic biological systems. Kadarmideen et al. (2006) introduced the concept of SG to animal science, highlighting that it could combine the already established GWAS methods with information about gene expression and metabolic pathways. Since then, SG has been crucial in understanding the biological role of genetic variants and genes identified with GWAS. Additionally, understanding of how genomic regions contribute to the variance of complex traits might help in selection of candidate markers; however, this has not been sufficiently studied so far. Using high-density single nucleotide polymorphism (SNP) chips, Koufariotis et al. (2014) found that SNPs in the upstream and downstream

Genetics and Molecular Research 15 (4): gmr15048930 
regions of genes in cattle were enriched for complex traits. Do et al. (2015) found that in pigs, SNPs in different annotated genomic regions contributed almost equally to the total genomic variance. However, their study was based on relatively few markers $(30 \mathrm{~K})$ and was performed in a population with high linkage disequilibrium (LD).

SG has been used to search for genes and biological processes involved in the regulation of cattle production traits, providing insights on biological processes associated with feed intake, feed efficiency, and weight gain. However, as of date, these biological processes are not well described for Bos indicus cattle. To enhance the understanding of the genetics that defines the production efficiency of Nellore cattle, we performed GWAS. Herein, we discuss the outcomes of our study with regard to feed intake, feed efficiency, and weight gain, within the SG framework. We investigated genetic variants, genomic variance partitioning, and physiological processes determined by the genomic regions mapped for these phenotypes.

\section{MATERIAL AND METHODS}

\section{Ethical statement}

No approval from the local Ethics Committee was required for the present study because we used the data previously collected in other experiments. In all the experiments, animals were handled in accordance with the guidelines of the Brazilian College of Animal Experimentation (COBEA). Phenotypic data and DNA samples were taken from each of the experiments, which had been approved by the respective Ethics Committees for each study (Santana et al., 2012; Gomes et al., 2013; Alexandre et al., 2015).

\section{Phenotypic data}

We collected data for 1334 Nellore bulls and steers $(484 \pm 95$ days old and $357 \pm$ $60.34 \mathrm{~kg}$ ) from 16 experiments conducted in Brazil from 2007 to 2013. These trials were carried out in individual pens with either GrowSafe or Calan Gates for recording the feed intake. Further details about the animals, phenotypes, facilities, experimental diets, and management procedures are available in Santana et al. (2012), Gomes et al. (2013), and Alexandre et al. (2015).

The animals were weighed every 21 days. Average daily gain (ADG) was estimated by linear regression of weight measurements over time. Dry matter intake (DMI) was recorded daily, and feed conversion ratio (FCR) was calculated by dividing DMI by ADG. Residual feed intake (RFI) was assumed to be the residual of the DMI regression on ADG, mid-body weight, and contemporary group. ADG, DMI, FCR, and RFI were tested for normality (Shapiro-Wilk) and homoscedasticity of residuals (Breusch-Pagan). Data points with standard deviations of \pm 3 from the mean were excluded from the phenotypic dataset.

\section{Genomic data and imputation}

Animals with phenotypic records were genotyped using three different commercially available panels: 388 with Illumina BovineHD ${ }^{\circledR}$ BeadChip (777,962 SNPs), 636 with GGP Indicus Neogen $\mathrm{HD}^{\circledR}$ (84,379 SNPs), and 310 with Illumina BovineSNP50 ${ }^{\circledR}$ version 2 BeadChip (54,609 SNPs). Only the genotype calls (standard cluster quality) greater than 0.70

Genetics and Molecular Research 15 (4): gmr15048930 
and samples with a call rate higher than $90 \%$ were kept in the dataset. Duplicate samples, ascertained when more than $95 \%$ alleles were identical by state based on 20,000 randomly obtained markers, were excluded from further analysis.

Genotypic information of 279 Nellore cattle genotyped with Illumina BovineHD ${ }^{\circledR}$ BeadChip and Affymetrix Axiom ${ }^{\circledR}$ Genome-Wide BOS 1 (648,874 SNPs) were combined and 1,261,128 SNPs were used. Imputation of the missing genotypes in the low-density SNP panel was performed using FImpute v2.2 (Sargolzaei et al., 2014) for the animals with phenotypic records. The accuracy of imputed genotypes was tested by cross-validation for all the procedures and concordance rate between the real and imputed genotypes was always higher than $97.5 \%$. As is the standard practice, genotypic data were submitted to quality control SNPs with minor allele frequency $<2 \%$, call rate $<95 \%$, or a significant deviation from Hardy-Weinberg equilibrium (Fisher exact test $\mathrm{P}$ value $<1 \times 10^{-5}$ ), and those that are located on allosomes were excluded. After imputation and quality control, 943,577 SNPs from 1334 phenotyped animals remained in the final dataset for the association test.

\section{Association analysis}

Considering the challenges of the "small n, large P" problem in GWAS, we used Lasso procedure implemented with a Gibbs sampler for parameter estimation within a Bayesian hierarchical framework, as described by Li et al. (2011). This approach considers covariate effects and proposes a more general penalization of the gene effect allowing sparsity, which means that the estimates of lower importance tended towards zero. The Gibbs sampler also estimates tuning parameters for the Lasso penalties, and thus, the degree of shrinkage of the marker effect is obtained from the data. A prior gamma distribution is assigned to the Lasso parameters and the conditional prior of the additive gene effect is assumed to follow a Laplace distribution.

ADG, DMI, FCR, and RFI were adjusted for contemporary groups (from the same experiment, fed the same diet, and administered under same management and environment) before the association test and the population substructures were evaluated. The animal's age was considered as a potential covariate for adjusting the responses, except for RFI, previous analysis of which did not show any correlation with age. We first filtered out the SNP database by performing GWAS with the single-SNP analysis described by Das et al. (2011), and only those markers with $\mathrm{P}$ value $\leq 0.1$ for the trait were selected for performing the Lasso procedure. Subsequently, the SNPs that passed through this last variable selection step were resubmitted to the GWAS method to refit the parameters in order to get lower biased estimates. We set a piecewise ratio (i.e., ratio of grouping SNPs) equal to three in order to obtain samples from the joint posterior distributions of additive gene effect with 5000 Markov chain Monte Carlo (MCMC) iterations after convergence, which was monitored by a potential scale reduction factor. Convergence was assumed to have been achieved when this factor was lower than 1.1. For claiming significance of the gene effects, the $95 \%$ posterior credible interval should not be zero. As a criterion for selecting SNPs with significant effects that were more relevant for the phenotype, we used the heritability estimate (i.e., portion of explained phenotypic variance) computed for each marker by using the posterior samples of the additive gene effect as recommended by Li et al. (2011). All computations and statistical analyses were performed using the gwas.lasso package for R, which is available at http://www.psu.edu/dept/statgen/ software.html.

Genetics and Molecular Research 15 (4): gmr15048930 


\section{Genomic variance partitioning}

The Ensembl variant effect predictor was used to annotate SNPs obtained by the Lasso procedure; five classes were used: downstream (variants found $5 \mathrm{~kb}$ downstream of a gene), exonic (variants found in coding region), intergenic (variants that occurred in-between genes), intronic, and upstream (variants found $5 \mathrm{~kb}$ upstream of a transcription start site). The total genomic variance of each annotation group was computed according to the gbayz function using the BayZ package (http://www.bayz.biz/). Briefly, the Bayesian Lasso model was the same as described above, except that 200,000 MCMC iterations were run with the first 20,000 as burn-in cycles. The gbayz function uses allelic effects from every 100 round of the MCMC and combines them to compute genomic estimated breeding values (GEBVs). The genomic variance was computed as the variance of GEBVs (Do et al., 2015). The total genomic variance was then computed according to the selected SNPs for a specific class of annotation as described above.

\section{Systems genetics analysis}

The gene set associated with each trait was determined from the Ensembl Genes 81 Database using the Biomart software by selecting genes located within $50 \mathrm{~kb}$ of each SNPflanking region with percentage of explained genomic variance higher than $1 \%$. Functional annotation of the gene sets was performed using the Database for Annotation, Visualization and Integrated Discovery (DAVID) v6.7 (Huang et al., 2009) against a bovine background (Bos taurus). Metabolic pathway analyses were based on the data available from the Kyoto Encyclopedia of Genes and Genomes (KEGG). To determine KEGG pathways significantly associated with all the traits, we implemented the Fisher exact test for assessing whether the genes in the gene set for each trait were overrepresented among all the genes in any given pathway.

\section{RESULTS}

\section{Association analysis}

ADG was associated with significant SNPs on chromosomes 1, 5, 6, 9, and 29. For DMI, chromosomes 2, 9, 19, 24, and 27 harbored significant SNPs. Among the feed efficiency traits, significant SNPs were observed on chromosomes 3, 4, 6, 7, 12, and 16 for FCR and on chromosomes $2,3,5,15$, and 22 for RFI (Table 1). The most associated genomic regions accounted for 14.67, $13.88,17.55$, and $16.83 \%$ of the genomic variance for ADG, DMI, FCR, and RFI, respectively.

The SNPs, arranged in order of their heritability rate, i.e., the portion of explained genomic variance, are shown in Table 1. Heritability and genomic variance estimates appear to be inflated, and must, therefore, be interpreted with caution. This was expected because only a small subset of SNPs was included in the final statistical procedure (Hoti and Sillanpää, 2006). Here, the heritability computation was used only as a metric for sorting significant SNPs by their relative importance to the phenotype.

\section{Genomic variance partitioning}

Five different classes were annotated for SNPs; the mean was $63.74 \%$ in the intergenic regions and $28.17 \%$ in the intron (Table 2). In general, the contribution of different annotated classes and the number of SNPs in these classes was linearly associated.

Genetics and Molecular Research 15 (4): gmr15048930 
Table 1. Single nucleotide polymorphisms that explain more than $1 \%$ genomic variance in feed efficiencyrelated traits.

\begin{tabular}{l|c|c|c|c}
\hline Trait & Chromosome & Position (bp) & Genomic variance (\%) & Heritability \\
\hline ADG & 1 & $52,750,864$ & 3.76 & 12.55 \\
\hline & 9 & $16,739,645$ & 3.56 & 11.85 \\
\hline & 5 & $107,376,496$ & 3.22 & 10.73 \\
\hline & 6 & $114,633,268$ & 1.07 & 3.22 \\
\hline DMI & 29 & $32,721,434$ & 7.11 & 19.92 \\
\hline & 24 & $50,506,180$ & 1.58 & 4.42 \\
\hline & 9 & $62,186,205$ & 1.46 & 4.09 \\
\hline & 27 & $17,684,976$ & 1.44 & 3.04 \\
\hline FCR & 9 & $66,777,623$ & 1.28 & 2.58 \\
\hline & 2 & $129,325,248$ & 1.01 & 14.10 \\
\hline & 19 & $32,867,556$ & 6.58 & 5.93 \\
\hline & 12 & $65,706,808$ & 2.77 & 4.35 \\
\hline & 6 & $2,084,530$ & 2.03 & 3.64 \\
\hline & 7 & $95,934,150$ & 1.70 & 2.56 \\
\hline & 4 & $68,743,352$ & 1.20 & 2.55 \\
\hline & 3 & $45,818,493$ & 1.19 & 2.30 \\
\hline & 3 & $45,812,332$ & 1.07 & 2.18 \\
\hline & 16 & $24,419,449$ & 8.67 & 15.77 \\
\hline & 16 & $24,345,227$ & 3.63 & 6.60 \\
\hline
\end{tabular}

$\mathrm{ADG}=$ average daily gain, $\mathrm{DMI}=$ dry matter intake, $\mathrm{FCR}=$ feed conversion ratio, $\mathrm{RFI}=$ residual feed intake.

Table 2. Percentage variance in different genomic regions, grouped by feed efficiency-related traits in Nellore
cattle.
\begin{tabular}{l|c|c|c|c|c}
\hline Genomic region & ADG & DMI & FCR & RFI & Mean \\
\hline Downstream & 3.30 & 3.22 & 3.20 & 3.47 & 3.30 \\
\hline Exon & 2.81 & 1.20 & 1.22 & 0.64 & 1.46 \\
\hline Intergenic & 62.49 & 64.71 & 64.74 & 63.02 & 63.74 \\
\hline Intron & 28.15 & 27.67 & 27.66 & 29.20 & 28.17 \\
\hline Upstream & 3.25 & 3.20 & 3.18 & 3.67 & 3.33 \\
\hline All & 100.0 & 100.0 & 100.0 & 100.0 & 100.0 \\
\hline
\end{tabular}

$\mathrm{ADG}=$ average daily gain, $\mathrm{DMI}=$ dry matter intake, $\mathrm{FCR}=$ feed conversion ratio, $\mathrm{RFI}=$ residual feed intake.

As expected, intergenic variants were the most common annotated variants. Similar results were reported in dairy and beef cattle by Koufariotis et al. (2014), who annotated $67.8 \%$ intergenic SNPs using Illumina BovineHD ${ }^{\circledR}$ BeadChip. These results are consistent with previous reports by Do et al. (2015), who observed that intergenic regions contributed to approximately $61-65 \%$ of the total genomic variance, depending on the traits. The authors suggested that LD among SNPs, poor functional annotation, and limitation in the number of SNPs (only 30K SNPs in their case) might affect the annotation results. In this study, we used high-density SNP panels; therefore, it is unlikely that SNP density was a major reason for the linear association reported above. The LD in cattle is also lower than that found in pigs.

Genetics and Molecular Research 15 (4): gmr15048930 


\section{Systems genetics}

The 50-kb criterium for selecting the flanking regions around significant SNPs was based on the known extent of LD blocks in cattle populations. A recent study on LD structure by Villa-Angulo et al. (2009) revealed that there were regions of high LD extending up to 100 $\mathrm{kb}$ and the size of haplotype blocks ranged between 30 bases and $75 \mathrm{~kb}$ (the average being 10.3 $\mathrm{kb}$ ). Previous studies also suggested that a similar distance could be used in the SG approach to capture the causal genes/SNPs affecting a quantitative trait like feed efficiency (Do et al., 2014). The over-representation analyses identified five KEGG pathways associated with feed efficiency-related traits (Table 3).

Table 3. Pathways involved in enrichment analysis of feed intake, efficiency, and performance traits in Nellore
cattle, obtained from KEGG.
\begin{tabular}{l|c|c|c}
\hline Pathway & Frequency in Geneset & Frequency in genome & P value \\
\hline Aldosterone-regulated sodium reabsorption & 3 & 39 & 0.006 \\
\hline T cell receptor signaling pathway & 3 & 106 & 0.011 \\
\hline Systemic lupus erythematosus & 4 & 25 & 0.018 \\
\hline Ion transporter inhibitors & 7 & 41 & 0.027 \\
\hline Proteoglycans & 3 & 222 & 0.045 \\
\hline
\end{tabular}

\section{DISCUSSION}

One of the main dilemmas of GWAS with high-density panels of genetic markers is addressing the problem of the "small n, large P", which is the observed scenario when the number of predictors far exceeds the sample size. This problem exists because not only such high dimensionality requires appropriate modeling for stable computations but also because of the fact that the more SNP markers we take into account, the greater is the need to control false positives. To overcome these challenges in the association analysis, we used Lasso procedure implemented with a Gibbs sampler for parameter estimation within a Bayesian hierarchical framework, as proposed by Li et al. (2011). Although more computationally expensive, this approach has advantages over methods based solely on single-SNP analysis in terms of parameter estimates.

Before variable selection, we pre-selected the markers with non-negligible effects on the trait by conducting a single-SNP analysis. We chose the cut-off criteria $\mathrm{P}$ value $<0.1$ to keep at least $10 \%$ of the full genotypic dataset for further analyses. Feed efficiency-related traits are complex and polygenic traits; they do not follow the Mendelian model of inheritance and are affected by several molecular processes with many potential influences, mainly the environmental factors. In ADG, $10.31 \%$ of all markers were selected for inclusion in the Lasso step, and 11.53, 11.79, and 13\% were selected for DMI, FCR, and RFI, respectively. In an attempt to identify the SNPs that would affect the traits, we applied a variable selection approach where the Lasso penalization was placed on the size of the additive effect. We set a piecewise ratio equal to three because it yielded more biologically relevant estimates without affecting the detection power when compared to computations that used a ratio of two. The animal's age at slaughter was treated as a covariate for ADG, DMI, and FCR, but it was significant only for ADG. All the SNPs selected by the Lasso procedure for ADG (588), DMI (604), FCR (559), and RFI (752) were resubmitted to the Bayesian model, but without imposing penalizations.

Genetics and Molecular Research 15 (4): gmr15048930 
This approach has the potential to improve the bias of parameter estimates (Li et al., 2011).

\section{Genomic variance partitioning}

The partitioning of genomic regions implies diversity in regulation of complex traits. Koufariotis et al. (2014) showed that, in cattle, the regulatory regions were significant for complex traits and the regions near genes might explain the high variance in human height and body mass index (Yang et al., 2011). The SNPs farther from the transcription start site were less likely to be significantly associated with the trait (Kindt et al., 2013). However, the annotation depends on many other factors. Perhaps, a common pattern for genomic variance does not exist; it depends on the biology of the traits, the population structure, and the LD among SNPs as well as on the relationship among the animals. The genomic partitioning, considering the LD among SNPs, and the partitioning based on the biological class might provide better insights.

\section{Systems genetics}

Aldosterone-regulated sodium reabsorption is associated with mineral reabsorption, specifically the renal sodium that is regulated by aldosterone from the adrenal glands. These physiological processes (mineral reabsorption) are energetically expensive and account for $10 \%$ of the maintenance body energy (Kies et al., 2005) and expenditure and could regulate the feed efficiency indirectly.

The T-cell receptors are related to cell fate and regulate the cell-mediated immunity. Apparently, these two mechanisms are not directly linked to the phenotypes studied here. However, T-cell receptor genes were observed to be upregulated in adipose tissue of pig lines that differed in feed efficiency (Lkhagvadorj et al., 2010) and were associated with RFI in Angus cattle (Rolf et al., 2012).

The molecular relationship between systemic lupus erythematosus (SLE) and the phenotypes studied here is not directly understandable. An SNP in the STAT4 gene was considered a genetic marker for fat metabolism in dairy cattle (Zhang et al., 2010) and this SNP was also related to SLE in humans (Ji et al., 2010). Interestingly, the protein encoded by the STAT family of genes is related to growth factors and the STAT6 gene polymorphisms were associated with growth efficiency and carcass traits in beef cattle (Rincon et al., 2009).

Previously, proteoglycans were reported to be associated with feed intake. The syndecan-3 (cell surface proteoglycan, mainly receptors) can influence feed intake (appetite) by the expression of MC3R and MC4R on hypothalamic neurons (Gantz and Fong, 2003). Therefore, other proteoglycans like hyaluronan, glypican, perlecan, and syndecans could also be related to feed intake.

The relationship between feed efficiency traits and the ion transport has been described in several studies (Richardson et al., 2004; Moore et al., 2009) and ion transport has been shown to contribute to the physiological variation of RFI in beef cattle (Richardson and Herd, 2004). Specifically, the $\mathrm{Na}^{+} / \mathrm{K}^{+}$-ATPase metabolism, which is related to homeostasis, can contribute up to $12 \%$ of the total body energy expenditure in cattle (McBride and Kelly, 1990). Homeostasis requires more energy expenditure for animal maintenance and it involves a series of metabolic pathways, mainly related to ion transport, energy metabolism, and protein turnover (Richardson et al., 2004). In addition, GWAS have also pointed to ion transport as 
one of the main mechanisms related to feed efficiency (Serão et al., 2013; Santana et al., 2014); global expression profile analysis in high and low feed efficiency animals showed a regulator of ion transport as one of the differentially expressed genes in Angus cattle (Chen et al., 2011).

\section{CONCLUSIONS}

Bayesian Lasso approach for GWAS, as adopted here, uncovered the genomic regions with biologically relevant genes and pathways for feed intake, feed efficiency, and performance. Metabolic pathway analysis using the SG approach may provide additional perspectives on the metabolic processes related to feed efficiency-related traits. Moreover, it also reinforces the earlier studies that indicated that ion transport is one of the most important physiological processes for the variation of these traits in beef cattle. These metabolic pathways are mainly related to the energy expended on maintenance of the ionic transport, body composition, and possible regulatory appetite processes. The genomic variance effected by the SNPs in the genome-wide association study was low; most of this variance was in the intergenic and intronic regions. These results could help in the genetic improvement of beef feed efficiency.

\section{Conflicts of interest}

The authors declare no conflict of interest.

\section{ACKNOWLEDGMENTS}

The contributions of Núcleo de Criadores de Nelore do Norte do Paraná, Luciano Borges (Rancho da Matinha), and Eduardo Penteado Cardoso (Fazenda Mundo Novo) are gratefully acknowledged. We would like to thank Dr. Zhong Wang for help with the gwas. lasso package. Research supported in part by São Paulo Research Foundation (FAPESP, \#2012/02039-9, \#2013/26902-0, \#2014/14121-7, \#2013/20571-2, and \#2014/07566-2) and Conselho Nacional de Desenvolvimento Científico e Tecnológico (CNPq, \#473249/2013-8 and \#442345/2014-3).

\section{REFERENCES}

Alexandre PA, Kogelman LJA, Santana MHA, Passarelli D, et al. (2015). Liver transcriptomic networks reveal main biological processes associated with feed efficiency in beef cattle. BMC Genomics 16: 1073. http://dx.doi. org/10.1186/s12864-015-2292-8

Anderson RV, Rasby RJ, Klopfenstein TJ and RT Clark. (2005). An evaluation of production and economic efficiency of two beef systems from calving to slaughter. J. Anim. Sci. 83: W 694-704.

Chen Y, Gondro C, Quinn K, Herd RM, et al. (2011). Global gene expression profiling reveals genes expressed differentially in cattle with high and low residual feed intake. Anim. Genet. 42: 475-490. http://dx.doi.org/10.1111/ j.1365-2052.2011.02182.x

Das K, Li J, Wang Z, Tong C, et al. (2011). A dynamic model for genome-wide association studies. Hum. Genet. 129: 629-639. http://dx.doi.org/10.1007/s00439-011-0960-6

Do DN, Ostersen T, Strathe AB, Mark T, et al. (2014). Genome-wide association and systems genetic analyses of residual feed intake, daily feed consumption, backfat and weight gain in pigs. BMC Genet. 15: 27. http://dx.doi. org/10.1186/1471-2156-15-27

Do DN, Janss LLG, Jensen J and Kadarmideen HN (2015). SNP annotation-based whole genomic prediction and selection: an application to feed efficiency and its component traits in pigs. J. Anim. Sci. 93: 2056-2063. http://dx.doi.

Genetics and Molecular Research 15 (4): gmr15048930 
org/10.2527/jas.2014-8640

Gantz I and Fong TM (2003). The melanocortin system. Am. J. Physiol. Endocrinol. Metab. 284: E468-E474. http:// dx.doi.org/10.1152/ajpendo.00434.2002

Gomes RC, Silva SL, Carvalho ME, Rezende FM, et al. (2013). Protein synthesis and degradation gene SNPs related to feed intake, feed efficiency, growth, and ultrasound carcass traits in Nellore cattle. Genet. Mol. Res. 12: 2923-2936. http://dx.doi.org/10.4238/2013.August.12.8

Havlík P, Valin H, Herrero M, Obersteiner M, et al. (2014). Climate change mitigation through livestock system transitions. Proc. Natl. Acad. Sci. USA 111: 3709-3714. http://dx.doi.org/10.1073/pnas.1308044111

Hoti F and Sillanpää MJ (2006). Bayesian mapping of genotype $\mathrm{x}$ expression interactions in quantitative and qualitative traits. Heredity 97: 4-18. http://dx.doi.org/10.1038/sj.hdy.6800817

Huang W, Sherman BT and Lempicki RA (2009). Systematic and integrative analysis of large gene lists using DAVID bioinformatics resources. Nat. Protoc. 4: 44-57. http://dx.doi.org/10.1038/nprot.2008.211

Ji JD, Lee WJ, Kong KA, Woo JH, et al. (2010). Association of STAT4 polymorphism with rheumatoid arthritis and systemic lupus erythematosus: a meta-analysis. Mol. Biol. Rep. 37: 141-147. http://dx.doi.org/10.1007/s11033-009-9553-z

Kadarmideen HN, von Rohr P and Janss LLG (2006). From genetical genomics to systems genetics: potential applications in quantitative genomics and animal breeding. Mamm. Genome 17: 548-564. http://dx.doi.org/10.1007/s00335-005-0169-x

Kies AK, Gerrits WJ, Schrama JW, Heetkamp MJ, et al. (2005). Mineral absorption and excretion as affected by microbial phytase, and their effect on energy metabolism in young piglets. $J$. Nutr. 135: 1131-1138.

Kindt ASD, Navarro P, Semple CA and Haley CS (2013). The genomic signature of trait-associated variants. BMC Genomics 14: 108. http://dx.doi.org/10.1186/1471-2164-14-108

Koufariotis L, Chen YP, Bolormaa S and Hayes BJ (2014). Regulatory and coding genome regions are enriched for trait associated variants in dairy and beef cattle. BMC Genomics 15: 436. http://dx.doi.org/10.1186/1471-2164-15-436

Li J, Das K, Fu G, Li R, et al. (2011). The Bayesian lasso for genome-wide association studies. Bioinformatics 27: 516523. http://dx.doi.org/10.1093/bioinformatics/btq688

Lkhagvadorj S, Qu L, Cai W, Couture OP, et al. (2010). Gene expression profiling of the short-term adaptive response to acute caloric restriction in liver and adipose tissues of pigs differing in feed efficiency. Am. J. Physiol. Regul. Integr. Comp. Physiol. 298: R494-R507. http://dx.doi.org/10.1152/ajpregu.00632.2009

McBride BW and Kelly JM (1990). Energy cost of absorption and metabolism in the ruminant gastrointestinal tract and liver: a review. J. Anim. Sci. 68: 2997-3010. http://dx.doi.org/10.2527/1990.6892997x

Moore SS, Mujibi FD and Sherman EL (2009). Molecular basis for residual feed intake in beef cattle. J. Anim. Sci. 87 (Suppl): E41-E47. http://dx.doi.org/10.2527/jas.2008-1418

Nkrumah JD, Basarab JA, Wang Z, Li C, et al. (2007). Genetic and phenotypic relationships of feed intake and measures of efficiency with growth and carcass merit of beef cattle. J. Anim. Sci. 85: 2711-2720. http://dx.doi.org/10.2527/jas.2006-767

Richardson EC and Herd RM (2004). Biological basis for variation in residual feed intake in beef cattle. 2. Synthesis of results following divergent selection. Aust. J. Exp. Agric. 44: 431-440. http://dx.doi.org/10.1071/EA02221

Richardson EC, Herd RM, Archer JA and Arthur PF (2004). Metabolic differences in Angus steers divergently selected for residual feed intake. Aust. J. Exp. Agric. 44: 441-452. http://dx.doi.org/10.1071/EA02219

Rincon G, Farber EA, Farber CR, Nkrumah JD, et al. (2009). Polymorphisms in the STAT6 gene and their association with carcass traits in feedlot cattle. Anim. Genet. 40: 878-882. http://dx.doi.org/10.1111/j.1365-2052.2009.01934.x

Rolf MM, Taylor JF, Schnabel RD, McKay SD, et al. (2012). Genome-wide association analysis for feed efficiency in Angus cattle. Anim. Genet. 43: 367-374. http://dx.doi.org/10.1111/j.1365-2052.2011.02273.x

Santana MHA, Rossi Junior P, Almeida R and Cucco DC (2012). Feed efficiency and its correlations with carcass traits measured by ultrasound in Nellore bulls. Livest. Sci. 145: 252-257. http://dx.doi.org/10.1016/j.livsci.2012.02.012

Santana MHA, Utsunomiya YT, Neves HHR, Gomes RC, et al. (2014). Genome-wide association analysis of feed intake and residual feed intake in Nellore cattle. BMC Genet. 15: 21. http://dx.doi.org/10.1186/1471-2156-15-21

Sargolzaei M, Chesnais JP and Schenkel FS (2014). A new approach for efficient genotype imputation using information from relatives. BMC Genomics 15: 478. http://dx.doi.org/10.1186/1471-2164-15-478

Serão NV, González-Peña D, Beever JE, Faulkner DB, et al. (2013). Single nucleotide polymorphisms and haplotypes associated with feed efficiency in beef cattle. BMC Genet. 14: 94. http://dx.doi.org/10.1186/1471-2156-14-94

Villa-Angulo R, Matukumalli LK, Gill CA, Choi J, et al. (2009). High-resolution haplotype block structure in the cattle genome. BMC Genet. 10: 19. http://dx.doi.org/10.1186/1471-2156-10-19

Yang J, Manolio TA, Pasquale LR, Boerwinkle E, et al. (2011). Genome partitioning of genetic variation for complex traits using common SNPs. Nat. Genet. 43: 519-525. http://dx.doi.org/10.1038/ng.823

Zhang F, Huang J, Li Q, Ju Z, et al. (2010). Novel single nucleotide polymorphisms (SNPs) of the bovine STAT4 gene and their associations with production traits in Chinese Holstein cattle. Afr. J. Biotechnol. 9: 4003-4008.

Genetics and Molecular Research 15 (4): gmr15048930 